TITLE:

\title{
Equilibrium and stationary nonequilibrium states in a chain of colliding harmonic oscillators
}

\section{$\operatorname{AUTHOR}(S)$ :}

Sano, Mitsusada M.

\section{CITATION:}

Sano, Mitsusada M.. Equilibrium and stationary nonequilibrium states in a chain of colliding harmonic oscillators. Physical Review E 2000, 61(2): 1144-1151

ISSUE DATE:

2000-02

URL:

http://hdl.handle.net/2433/59076

RIGHT:

Copyright 2000 American Physical Society 


\title{
Equilibrium and stationary nonequilibrium states in a chain of colliding harmonic oscillators
}

\author{
Mitsusada M. Sano \\ Department of Fundamental Sciences, Faculty of Integrated Human Studies, Kyoto University, Sakyo-ku, Kyoto, 606-8501, Japan
}

(Received 21 April 1999)

\begin{abstract}
Equilibrium and nonequilibrium properties of a chain of colliding harmonic oscillators (ding-dong model) are investigated. Our chain is modeled as harmonically bounded particles that can only interact with neighboring particles by hard-core interaction. Between the collisions, particles are just independent harmonic oscillators. We are especially interested in the stationary nonequilibrium state of the ding-dong model coupled with two stochastic heat reservoirs (not thermostated) at the ends, whose temperature is different. We check the Gallavotti-Cohen fluctuation theorem [G. Gallavoti and E. G. D. Cohen, Phys. Rev. Lett. 74, 2694 (1995)] and also the Evans-Searles identity [D. Evans and D. Searles, Phys. Rev. E. 50, 1994 (1994)] numerically. It is verified that the former theorem is satisfied for this system, although the system is not a thermostated system.
\end{abstract}

PACS number(s): 05.20. $-\mathrm{y}, 05.45 .-\mathrm{a}, 44.10 .+\mathrm{i}$

\section{INTRODUCTION}

For more than a decade, nonequilibrium statistical mechanics has been revived by many authors from the microscopic point of view. A vast literature related to this topic has been published. It was actually a fruitful revival. The main question there is, "How do the dynamical properties of a given system explain phenomenology derived from (non)equilibrium statistical mechanics?" A partial answer has been is obtained for a class of hyperbolic systems. For instance, the dynamical characterization of hydrodynamic transport properties, especially diffusion, is obtained for homogeneous chaotic systems using the escape rate formalism [1]. The second example is entropy production by defining the time evolution of the Gibbs entropy for a given system [2]. The phase space contraction plays an important role there. Another formulation of entropy production does not need the phase space contraction, but needs coarse graining and the thermodynamic limit [3]. A third example is motivated by the results of numerical simulation of nonequilibrium molecular dynamics (i.e., a thermostated system or Nosé-Hover system) [4]. Proposing a guiding principle (the so-called chaotic hypothesis), the fluctuation of a stationary nonequilibrium state is characterized by entropy production [4]. The derived theorem is named the Gallavotti-Cohen fluctuation theorem (the GCFT).

One experimental verification of the GCFT can be carried out for the heat conduction in a nonlinear lattice chain. Since the work of Fermi, Pasta, and Ulam (FPU), a number of models have been numerically investigated. Recently, an experimental verification of the GCFT has been done for a FPU $\beta$ chain with a thermostat [5]. Furthermore, stimulated by the work of [4], the uniqueness of a stationary nonequilibrium state and the existence of entropy production in stochastically driven nonlinear lattice chain have been rigorously proved [6]. In this paper, we treat a different model from [5], and additionally, under a different condition (stochastic boundary condition). More precisely, we investigate equilibrium and stationary nonequilibrium states in the ding-dong model coupled with a heat reservoir, not a thermostat, especially the Fourier law and entropy production. A detailed investigation of the Fourier law and the Green-Kubo relation of heat conduction for the ding-dong model is found in [7]. Our aim here is to verify the GCFT [4] and another theorem [the Evans-Searles (ES) identity] [8] which concerns the ensemble of initial conditions, for the ding-dong model with a heat reservoir, not a thermostat. As mentioned in [9], a mathematical proof of the GCFT for this type of system (dynamical system + stochastic heat reservoir) is beyond present mathematical ability. (See also [10] for a generalization to Langevin dynamics.) Thus we carry out a numerical computation. As a result, we present an example of the verification of the GCFT under the stochastic boundary condition.

The organization of this paper is as follows. In Sec. II, we introduce the ding-dong model. In Sec. III, the equilibrium property of the ding-dong model is examined numerically. In Sec. IV, the stationary nonequilibrium state of the ding-dong model is investigated. We consider heat conduction and entropy production. The GCFT and ES identity are checked numerically. In Sec. V, we summarize our conclusions.

\section{DING-DONG MODEL}

We introduce the ding-dong model [7] which was introduced as a simplified model of the ding-a-ling model [11] in the context of heat conduction. The ding-a-ling model consists of harmonic oscillators placed on a line with free particles. The free particles are placed between each neighboring harmonic oscillator. In this model, the interaction is the elastic collision between the neighboring free particle and the particle of the oscillator. Between collisions, the free particles and harmonic oscillators move independently. The ding-dong model is obtained by just removing all free particles in the ding-a-ling model. Thus, in this case, the neighboring harmonic oscillators can elastically collide with each other. It is not well known, but the ding-dong model is equivalent to Dawson's one-dimensional plasma sheet model [12-16]. Thus, the ding-dong model is never an artificial model, but a physical model. The Hamiltonian of the dingdong model is given as 


$$
H=\sum_{i=0}^{N} \frac{p_{i}^{2}}{2}+\frac{q_{i}^{2}}{2}
$$

with the elastic constraint

$$
q_{i+1}+1 \geqslant q_{i}
$$

$q_{i}$ represents the displacement from the $i$ th lattice point. For our convenience, we set the lattice constant to $a=1$. Particles move as harmonic oscillators around the lattice point, but can collide with the nearest neighbor particles to exchange energy. Some statistical properties of the ding-dong model have been investigated by several authors: Kitahara et al. [13-16] for the properties of a one-dimensional plasma and Prosen and Robnik [7] for heat conduction (the Fourier law) by extensive numerical calculation.

Here we comment on one point of the numerical aspects of the ding-dong model. Since particles are arranged in a one-dimensional line and collide with each other, this model has numerical merit. Almost the time of numerical time evolution is spent for the calculation of the next collision time. In this case, the use of the heap sort algorithm accelerates the numerical calculation.

Next we show the setup of time evolution of the dingdong model and explain approximately the behavior of the system, i.e., chaoticity. Detailed investigation of the dingdong model is found in [7]. First we note the following point. We investigate the statistical behavior of the ding-dong model. Thus we consider only two-body collisions, since it is expected that three-body and higher-body collisions are events with Lebesgue measure zero. Therefore, "collision", means a two-body collision hereafter. We denote as $t_{k}$ the time that the $k$ th collision occurs and $\left\{t_{k}\right\}_{k=-\infty}^{+\infty}$ is a set of collisions. Further, we set the intercollision time $\tau_{k}$ as

$$
\tau_{k}=t_{k}-t_{k-1}
$$

The collision is elastic. Then if the $i$ th particle collides with the $(i+1)$ th particle, after collision, their momenta are exchanged:

$$
\begin{aligned}
& p_{i}^{+}=p_{i+1}^{-}, \\
& p_{i+1}^{+}=p_{i}^{-},
\end{aligned}
$$

where $i=1,2, \ldots, N-1$. Between collisions, the system obeys the following Hamiltonian equation of motion:

$$
\frac{d}{d t} q_{i}=p_{i}, \quad \frac{d}{d t} p_{i}=-q_{i}
$$

The map from the $k$ th collision to the $(k+1)$ th collision can be constructed as

$$
\Phi=\Phi_{\text {collision }} \bigcirc \Phi_{\text {oscillator }}^{\tau\left(\mathbf{x}_{k}\right)}
$$

where $\mathbf{x}=\left(q_{1}, q_{2}, \ldots, q_{N}, p_{1}, p_{2}, \ldots, p_{N}\right)^{\top} . \tau(\mathbf{x})$ is the time that the next collision occurs starting from the point $\mathbf{x}$. $\tau(\mathbf{x})$ is called the ceiling function. $\mathbf{x}_{k}$ is the position and momentum at the $k$ th collision. $\Phi_{\text {collision }}$ for the collision between the $i$ th and the $(i+1)$ th particles is given as

$\Phi_{\text {oscillator }}^{\tau\left(\mathbf{x}_{k}\right)}$ is given as 


$$
\left(\begin{array}{c}
q_{1}\left(t_{k}+\tau\left(\mathbf{x}_{k}\right)\right) \\
q_{2}\left(t_{k}+\tau\left(\mathbf{x}_{k}\right)\right) \\
\vdots \\
q_{N}\left(t_{k}+\tau\left(\mathbf{x}_{k}\right)\right) \\
p_{1}\left(t_{k}+\tau\left(\mathbf{x}_{k}\right)\right) \\
p_{2}\left(t_{k}+\tau\left(\mathbf{x}_{k}\right)\right) \\
\vdots \\
p_{N}\left(t_{k}+\tau\left(\mathbf{x}_{k}\right)\right)
\end{array}\right)=\left(\begin{array}{c}
p_{1}\left(t_{k}\right) \sin \left[\tau\left(\mathbf{x}_{k}\right)\right]+q_{1}\left(t_{k}\right) \cos \left[\tau\left(\mathbf{x}_{k}\right)\right] \\
p_{2}\left(t_{k}\right) \sin \left[\tau\left(\mathbf{x}_{k}\right)\right]+q_{2}\left(t_{k}\right) \cos \left[\tau\left(\mathbf{x}_{k}\right)\right] \\
\vdots \\
p_{N}\left(t_{k}\right) \sin \left[\tau\left(\mathbf{x}_{k}\right)\right]+q_{N}\left(t_{k}\right) \cos \left[\tau\left(\mathbf{x}_{k}\right)\right] \\
p_{1}\left(t_{k}\right) \cos \left[\tau\left(\mathbf{x}_{k}\right)\right]-q_{1}\left(t_{k}\right) \sin \left[\tau\left(\mathbf{x}_{k}\right)\right] \\
p_{2}\left(t_{k}\right) \cos \left[\tau\left(\mathbf{x}_{k}\right)\right]-q_{2}\left(t_{k}\right) \sin \left[\tau\left(\mathbf{x}_{k}\right)\right] \\
\vdots \\
p_{N}\left(t_{k}\right) \cos \left[\tau\left(\mathbf{x}_{k}\right)\right]-q_{N}\left(t_{k}\right) \sin \left[\tau\left(\mathbf{x}_{k}\right)\right]
\end{array}\right),
$$

where $\mathbf{x}_{k}$ is the $k$ th collision point. With the condition that the center of mass is zero, if we carry out a certain canonical transformation,

$$
\mathbf{x}=(\mathbf{q}, \mathbf{p}) \mapsto \mathbf{X}=(\mathbf{Q}, \mathbf{P}),
$$

where $\mathbf{Q}=\left(Q_{1}, \ldots, Q_{N-1}\right), \mathbf{P}=\left(P_{1}, \ldots, P_{N-1}\right)$, then after diagonalization, we obtain the Hamiltonian finally:

$$
H=\frac{1}{2} \sum_{i=1}^{N-1}\left(\lambda_{i} Q_{i}^{2}+\mu_{i} P_{i}^{2}\right)=E,
$$

where the $\lambda_{i}$ 's and $\mu_{i}$ 's are determined by the diagonalization. The elastic constraint Eq. (2) now becomes

$$
\mathcal{F}^{(i)}(\mathbf{Q}) \equiv \sum_{k=1}^{N-1}\left(U_{i k}-U_{i+1 k}\right) Q_{k} \leqslant 1,
$$

where $i=1,2, \ldots, N-1$ and $U_{i k}$ is the matrix element for the diagonalization. The Hamiltonian is for an $(N-1)$-dimensional anisotropic harmonic oscillator with hard walls satisfying Eq. (12). We can check that the hard walls surround the stable equilibrium point at the origin. Hence if the total energy is sufficiently low, the system is integrable. Because the trajectory goes around the stable equilibrium point, there is a critical value of the total energy for chaos [7]. But when the total energy is high enough, the system behaves like an integrable system. In this case, the particle moves ballistically and collides with the walls defined in Eq. (12). Therefore, in the intermediate total energy range, the system exhibits chaos. From numerical calculation for a few-body system, in this range, the system seems to be nonhyperbolic [15]. But we expect that a chaotic sea dominates the phase space in this energy range of a system with a sufficiently large number of particles and the system has effectively an important requirement of the GCFT, i.e., the transitive Anosov property.

\section{EQUILIBRIUM PROPERTIES}

In this section, we show the equilibrium properties of the ding-dong model. First, in order to characterize how particles interact with each other, we calculate the intercollision time distribution. Numerical calculation was done for the total energy $E=120$ and the number of particles $N=100$. The result obtained is depicted in Fig. 1. The distribution shows good agreement with the exponential distribution

$$
P(\tau) d \tau=\alpha e^{-\alpha \tau} d \tau
$$

where $\alpha$ depends on the total energy and the number of particles. This implies that particles randomly collide with each other.

Second, we check the velocity distribution. Figure 2 shows the result starting from a certain random initial condition with the total energy $E=2500$ and the total number of particles $N=2500$ after 10000 collisions. The distribution well fits a Gaussian distribution, namely, the Maxwell distribution. Therefore, in some sense, the system is expected to behave as a gas system.

Third, we consider the heat current in the ding-dong model. We define the heat current at the $k$ th collision between the $i$ th and $(i+1)$ th particles:
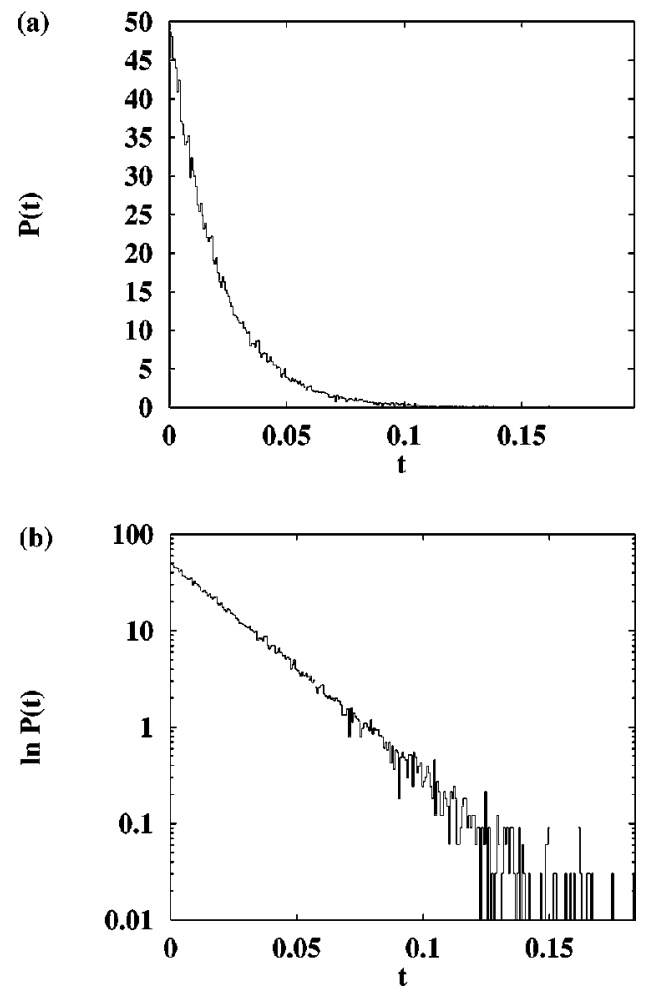

FIG. 1. Intercollision time distribution $N=100, E=120$. (a) The plot is obtained after $10^{5}$ collisions. (b) Log scale of (a). 


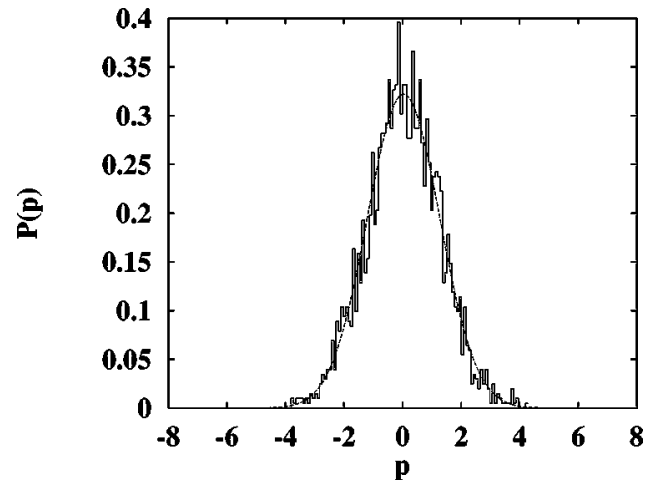

FIG. 2. Velocity distribution for $N=2500, E=2500$. Starting from a certain initial condition and after long time evolution, this plot is obtained. The dotted line is Gaussian fitting.

$$
j_{i}(t)=\frac{1}{2}\left(p_{i+1}-p_{i}\right)\left(p_{i+1}^{2}-p_{i}^{2}\right) \delta\left(q_{i+1}-q_{i}+1\right) \delta\left(t-t_{k}\right) .
$$

The heat current distribution is depicted in Fig. 3. The plot is obtained for $N=250, E=250$. The distribution has an unfamiliar form and is symmetric with respect to $j=0$.

These three distributions in the equilibrium state will be compared with those in the stationary nonequilibrium state in Sec. IV. In particular, we will remark the deviation from the equilibrium case in the stationary nonequilibrium state.

\section{STATIONARY NONEQUILIBRIUM STATE, THERMAL CONDUCTION, AND ENTROPY PRODUCTION}

In this section, we investigate the stationary nonequilibrium state of the ding-dong model, especially the Fourier law
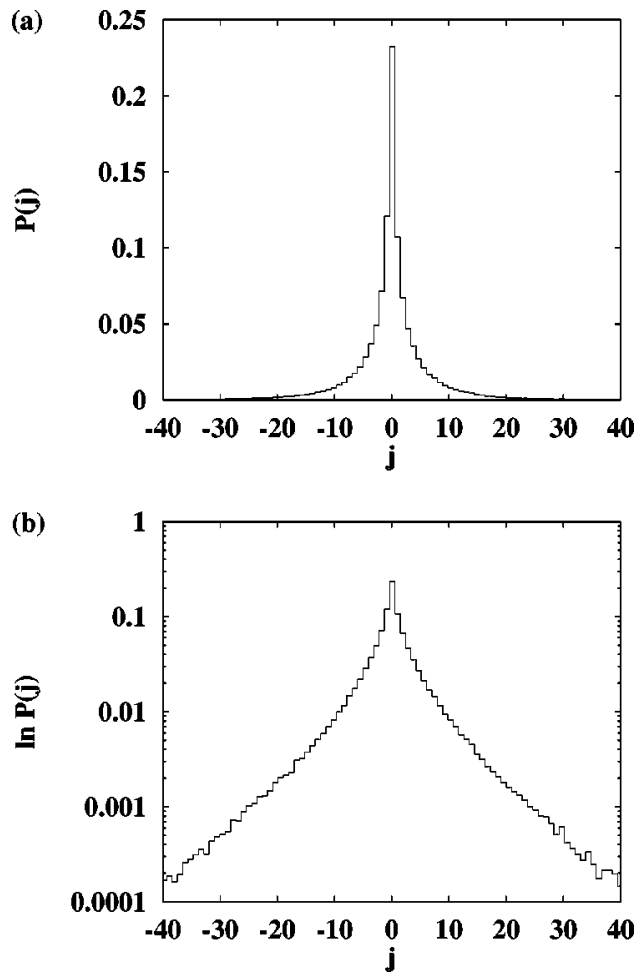

FIG. 3. Heat current distribution for $N=250, E=250$. (a) Normal scale. The plot is obtained after $10^{5}$ collisions. (b) Log scale of (a). and entropy production.

Before introducing the recent theory, we recall the following two important facts from thermodynamics. A system coupled to heat reservoirs having different temperature at the boundaries obeys the Fourier law

$$
\mathbf{j}=\kappa \boldsymbol{\nabla} T,
$$

where $\mathbf{j}$ is the heat current vector and $\kappa$ is the heat conductivity. The entropy production $\sigma(\mathbf{x})$ is defined as

$$
\sigma(\mathbf{x})=\mathbf{j} \cdot \nabla\left(\frac{1}{T}\right) .
$$

Thus the total entropy production $\Sigma$ of the domain with linear temperature gradient for a one-dimensional system is

$$
\Sigma=\int_{L}^{R} d x \sigma(x)=j\left(\frac{1}{T_{R}}-\frac{1}{T_{L}}\right),
$$

where $T_{L}$ and $T_{R}$ are the temperature of the left and right heat reservoirs, respectively. Later, this result will be compared with the numerical calculation of the GCFT and ES identity.

\section{A. Gallavotti-Cohen fluctuation theorem and Evans-Searles identity}

We here briefly summarize the GCFT [4] and the ES identity [8]. The original derivation was for two-dimensional shearing flow with a thermostat [4]. We assume that the system is chaotic. More precisely, $(A)$ the system is a transitive Anosov system. Further, we assume that $(B)$ the system is dissipative and $(C)$ the system is time-reversible. The entropy production is defined as the contraction rate of the phase space volume [2]. The condition $(B)$ implies that the entropy production is positive for this setting. Dynamical characterization of entropy production starts from the idea of Sinai, Ruelle, and Bowen (SRB), i.e., the construction of the SRB measure $\bar{\mu}$. The SRB measure can be constructed by the expansion coefficient of the Poincare map $S^{\tau}$. Here we consider the finite time average of the entropy production at $\mathbf{x}$ :

$$
\sigma_{\tau}(\mathbf{x})=\frac{1}{\tau} \sum_{j=-\tau / 2}^{\tau / 2-1} \sigma\left(S^{j} \mathbf{x}\right)=\langle\sigma\rangle a_{\tau}(\mathbf{x}),
$$

where $S$ is the Poincaré map. Fluctuation of the entropy production from its mean value is characterized by $a_{\tau}(\mathbf{x})$. We denote $\pi_{\tau}(p) d p=P\left(a_{\tau} \in(p, p+d p)\right)$ the probability measure for $a_{\tau}$. We consider the ratio of the probabilities $\pi_{\tau}(p) d p$ and $\pi_{\tau}(-p) d p$. The GCFT is the following relation:

$$
\frac{\pi_{\tau}(p)}{\pi_{\tau}(-p)}=e^{\tau\langle\sigma\rangle p}
$$

The proof is given by using the construction of the SRB measure (i.e., the expansion coefficient). For the detailed derivation, see [4]. This relation is considered to be the result of the large deviation property. In the next subsection, for the stationary nonequilibrium state of the ding-dong model, we 
check the linearity of $\ln \pi_{\tau}(p) / \pi_{\tau}(-p)$ in $p$. In our experiment, we do not use a thermostat, but a stochastic heat reservoir. Therefore, in the strict sense, the numerical experiments carried out in quite different conditions from those of the GCFT. As a dynamical system, the condition $(A)$ is effectively valid, and the condition $(B)$ is valid. But the condition $(C)$, i.e., time-reversality, should be replaced by the terminology of stochastic dynamics. On the other hand, the ES identity concerns the ensemble of initial conditions [8]:

$$
\frac{\mu_{L}\left(E_{p}\right)}{\mu_{L}\left(E_{-p}\right)}=e^{\tau\langle\sigma\rangle p},
$$

where $\mu_{L}$ represents the Liouville measure and $E_{p}$ is the set of initial conditions having the value $p$ of the observable. The formula is very similar to the GCFT. But the ES identity measures transient behavior toward the stationary state. We have to carefully distinguish them. The difference between the two theorems is explained in detail in [17].

\section{B. Numerical results}

Let us define the heat reservoir. We set the walls at the ends of the system. The other side of the wall is the heat reservoir. In the numerical calculation, we set the walls at the first lattice point minus 0.8 and at the $N$ th lattice point plus 0.8. All numerical calculation has been done in this setting. The momentum of particles in the heat reservoir is distributed according to the distribution

$$
P(p) d p=\frac{|p|}{T} \exp \left(-\frac{p^{2}}{2 T}\right) d p .
$$

For the left (right) reservoir, we take the plus (minus) sign of momentum. This setting of the heat reservoir is very important [18]. We set the temperature of the heat reservoir at the ends $T_{L}$ (left) and $T_{R}$ (right), respectively. When a particle at the ends $(i=1, N)$ collides with the wall, the particle instantaneously collides with a particle inside the heat reservoir. The temperature of the heat reservoir is set to $T_{L}=2.0$ and $T_{R}=1.5$, which were well checked the Fourier law in [7]. The local temperature $T_{k}$ around the $k$ th particle is defined as

$$
T_{k}=\left\langle p_{k}^{2}\right\rangle
$$

$\langle\cdots\rangle$ means the time average or phase space average. For numerical calculation, we use the time average.

First we check the Fourier law. Figure 4 is the temperature profile $\left(N=50, T_{L}=2.0, T_{R}=1.5\right)$. The end plots correspond to the temperature of the left and right heat reservoirs, respectively. There is an edge effect. The temperature of the end of the bulk at the left (right) is not $T_{L}\left(T_{R}\right)$, respectively. (This edge effect can be removed by the trick in [7]. We also used this trick and have done a few numerical calculation. The result of that is similar to that in this paper. Thus the edge effect does not change the conclusion of the verification of the GCFT.) Relaxation to the stationary nonequilibrium state is very slow. We check the stationary condition from the constancy of the time-averaged local current and temperature. The temperature profile is still a zigzag shape, not smooth, but we can see the linear dependence of the Fourier law.

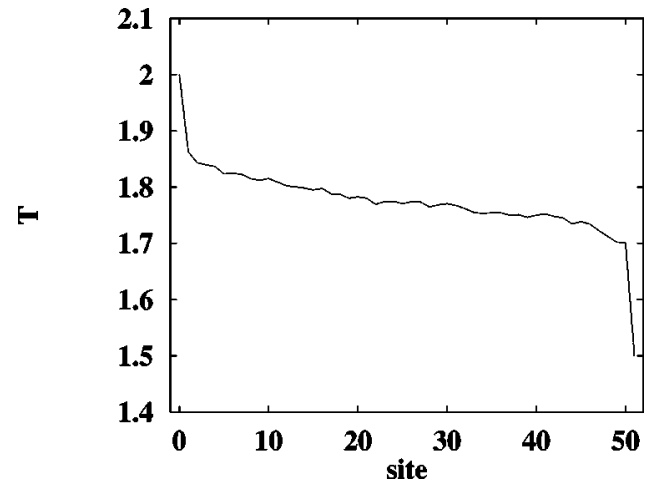

FIG. 4. Temperature distribution $N=50, T_{L}=2 \cdot 0, T_{R}=1.5$. The plot is obtained starting from a certain initial condition, and after $5 \times 10^{6}$ collisions. The end plots correspond to the temperatures of the left and right heat reservoirs.

In order to check the statistical difference between the equilibrium state and the stationary nonequilibrium state, we check three distributions investigated for equilibrium states in Sec. III. In Fig. 5, the distribution of intercollision time is depicted $\left(N=250, T_{L}=2.0, T_{R}=1.5\right)$. After $10^{5}$ collisions and $3 \times 10^{6}$ collisions, $10^{5}$ collisions are sampled. The mean intercollision time is on the order of $10^{-2}$ for this case. The distribution obtained well fits an exponential distribution. This shows that the collisions occur randomly. Compared with the equilibrium case, we cannot see a difference. But in the following two distributions, we can see the difference between the equilibrium state and the stationary nonequilibrium state.

In Fig. 6, the velocity distribution is shown. As expected, the distribution shows good agreement with a Gaussian dis-
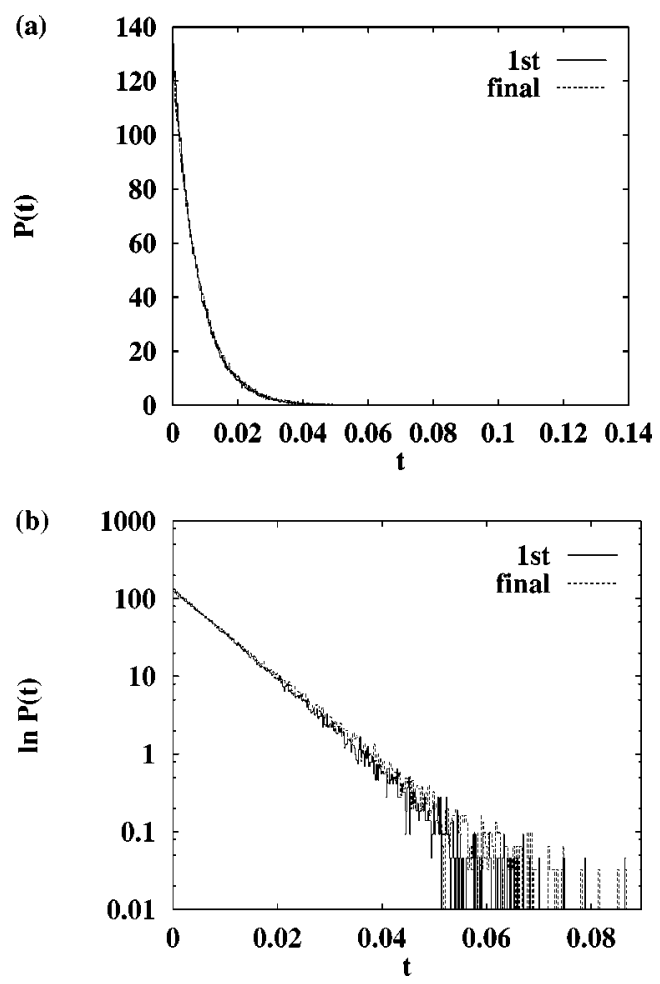

FIG. 5. Intercollision time distribution $N=250, T_{L}=2 \cdot 0, T_{R}$ $=1.5$. (a) The plot is obtained after $10^{5}$ collisions and $3 \times 10^{6}$ collisions. (b) Log scale of (a). 
(a)
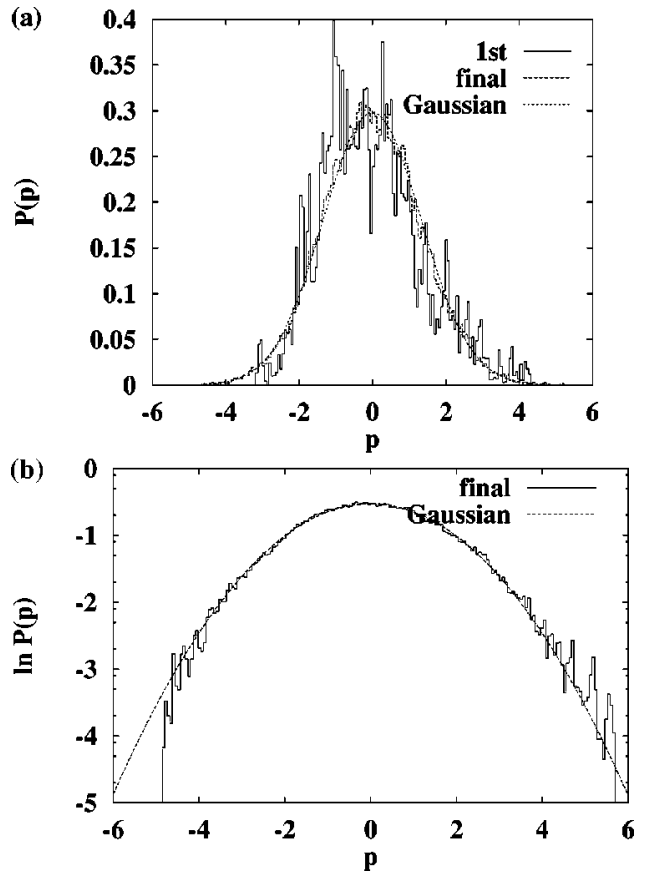

FIG. 6. Velocity distribution $N=250, T_{L}=2.0, T_{R}=1.5$. The plot is obtained starting from a certain initial condition, up to $10^{5}$ collisions and $3 \times 10^{6}$ collisions. (a) Initial ( $10^{5}$ collisions) and final $\left(3 \times 10^{6}\right.$ collisions) distribution. (b) Log scale. The distribution tends to a Gaussian distribution but the tail of the distribution is asymmetric.

tribution (i.e., the Maxwell distribution), but toward the stationary state, gradually the tail of the distribution becomes asymmetric. This asymmetry suggests energy transfer. This asymmetry is naturally observed in the heat current distribution.

Time variation of the heat current distribution is depicted in Fig. 7. Note that the distribution is not Gaussian as in the equilibrium state. After a long run, the tail of the distribution becomes asymmetric, which seems to be evidence of stationary energy transfer. We remark that in the stationary nonequilibrium state, the velocity distribution and heat current distribution are asymmetric in their tails. The stationary nonequilibrium state is close to the equilibrium state in some sense.

In Fig. 8(a), the spatial distribution of the time-averaged heat current $\bar{j}_{i, \tau}=(1 / \tau) \int_{t}^{t+\tau} j_{i}\left(t^{\prime}\right) d t^{\prime}$ is depicted. It was ob-

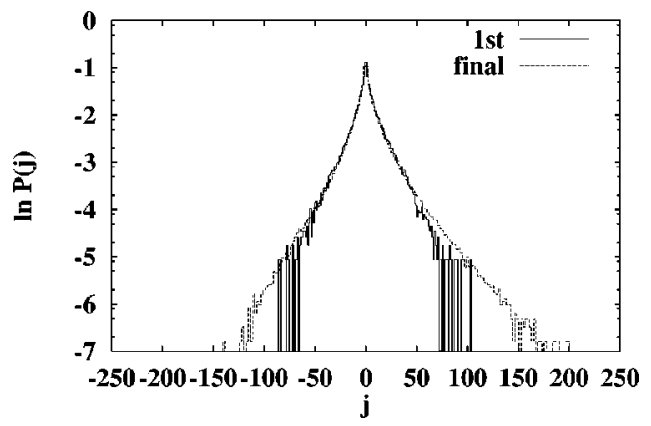

FIG. 7. Heat current distribution $N=250, T_{L}=2 \cdot 0, T_{R}=1.5$, starting from a certain initial condition, for $10^{5}$ collisions and 3 $\times 10^{6}$ collisions After sufficient time evolution, the tail of the distribution becomes asymmetric.
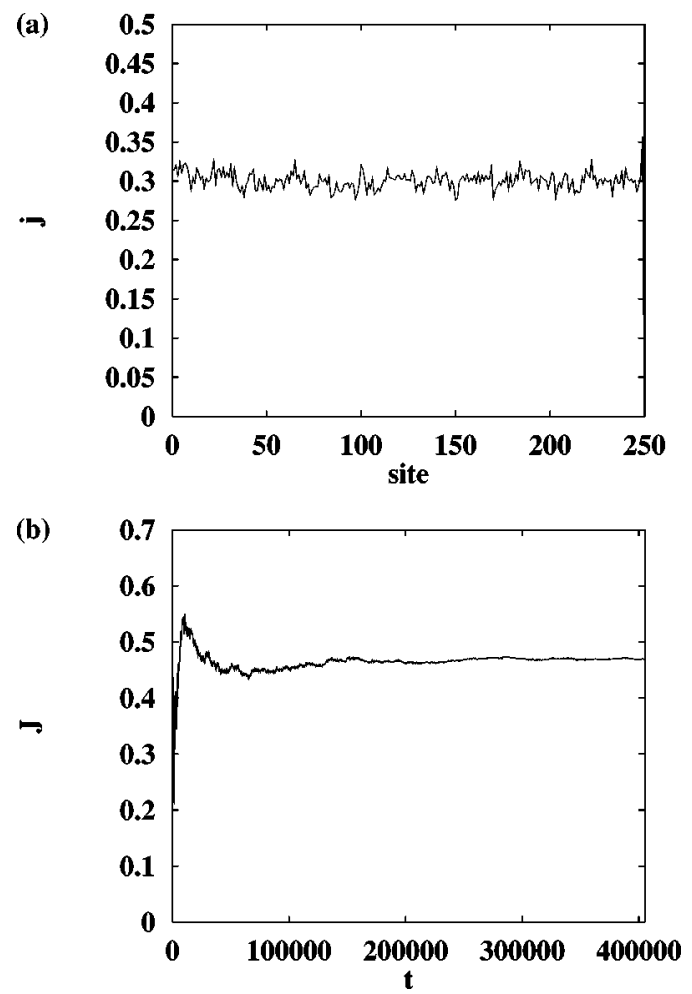

FIG. 8. Spatial distribution of heat current and relaxation of mean heat current (a) Spatial distribution of heat current that occurred up to $3 \times 10^{6}$ collisions starting from a certain initial configuration. $N=250, T_{L}=2 \cdot 0, T_{R}=1.5$ (b) Relaxation of mean heat current. $N=50, T_{L}=2.0, T_{R}=1.5$.

tained after $3 \times 10^{6}$ collisions starting from a certain initial condition. Although there is still fluctuation, the heat current is almost constant over all sites. In Fig. 8(b) the relaxation of the mean heat current $J_{\tau}=(1 / N) \sum_{i=1}^{N} \bar{j}_{i, \tau}$ is depicted for $N$ $=50$. The relaxation is very slow.

The entropy production can be checked by using Eq. (19) (the GCFT) and Eq. (20) (the ES identity). We define the distribution of $P_{\tau}(z)$ of the following quantity $z$ :

$$
z=\frac{J_{\tau}}{J_{\infty}}
$$

In this case, the GCFT becomes

$$
\ln \frac{P_{\tau}(z)}{P_{\tau}(-z)}=\tau z \Sigma=\tau z J_{\infty}\left(\frac{1}{T_{R}}-\frac{1}{T_{L}}\right) .
$$

We consider that the system does not possess a thermostat, but has a stochastic heat reservoir. Thus this relation is not so trivial for our system. It is worth testing Eq. (24) numerically.

First, we check the ES identity for our ding-dong model with a heat reservoir. In Fig. 9, we show the result $(N$ $\left.=100, T_{L}=2.0, T_{R}=1.5\right)$. In Fig. 9(a), the time evolution of the mean heat current distribution is depicted. Figure 9 (a) is 
(a)

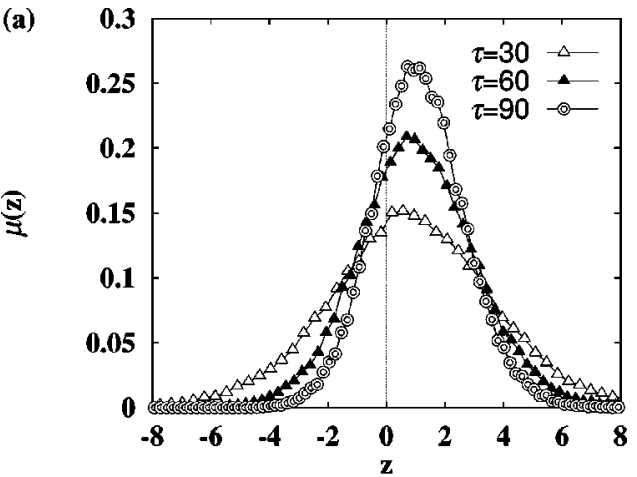

(b)

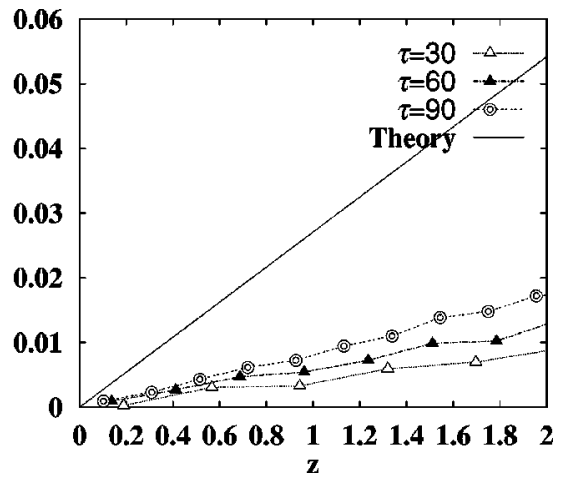

FIG. 9. Check of the ES identity. (a) Heat current distribution $\mu_{\tau}(z)(\tau=30,60,90)$ obtained from 50000 random initial conditions. (b) $J$ vs $(1 / \tau) \ln \left[\mu_{\tau}(z) / \mu_{\tau}(-z)\right], \quad \tau=30,60,90 . \quad N=100, T_{L}$ $=2.0, T_{R}=1.5$. The line labeled "Theory" corresponds to the theoretical slope $\Sigma$.

obtained from the time evolution of 50000 random initial configurations. The initial condition is prepared as follows. We checked and fixed the mean average of the total energy. With this total energy, the initial position and momentum are randomly sampled. It seems that the distribution tends to a limiting form obeying the central limit theorem. From numerical calculation, $J_{\infty}$ can be estimated as $J_{\infty} \approx 0.400$ and also $T_{R}^{\prime} \approx 1.66, T_{L}^{\prime} \approx 1.87$ (the temperatures of the particles $n=N$ and 1 , respectively). The entropy production is now $\Sigma \equiv J_{\infty}\left(1 / T_{R}^{\prime}-1 / T_{L}^{\prime}\right) \approx 0.0271$. Note that $\Sigma$ is obtained from the actually observed temperature slope of the bulk. In Fig. 9(b), we check the ES identity. We can see the linearity of $(1 / \tau) \ln [\mu(z) / \mu(-z)]$ in $z$ clearly, which suggests that the ES identity holds and the entropy production is nonzero. But the entropy production is below our theoretical prediction. Several reason can be considered: (1) the difficulty of preparation of the ensemble of initial conditions; (2) the system does not reach the stationary state; (3) the boundary effect; (4) the size effect, i.e., the thermodynamic limit. Among these possibilities, we believe that the reason is points (1) and (2), especially point (2). In order to check this, we are actually doing a long-time run. However, our CPU power is not sufficient for this numerical calculation (i.e., long time for the stationarity and ensemble average). It is estimated that it will take about two months on our machine, so this problem is reserved for future study.

The GCFT can be checked by long-time run of one trajectory toward the stationary nonequilibrium state. In Fig. 10, we depict the numerical result. Figure 10(a) is obtained
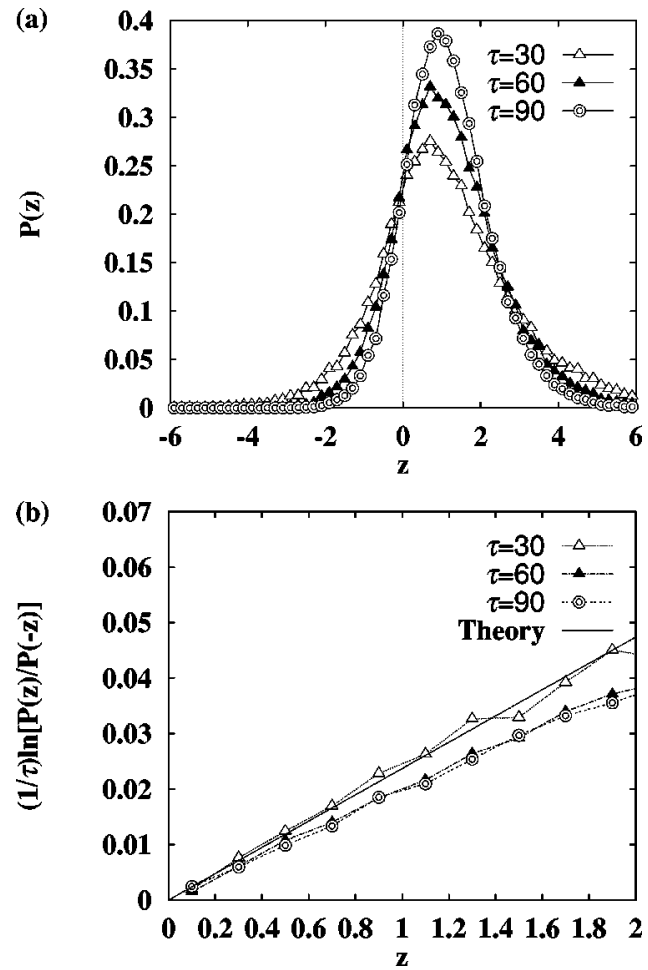

FIG. 10. Check of the GCFT. (a) Heat current distribution $P_{\tau}(z)$ $(\tau=30,60,90)$ obtained from a given trajectory. (b) $J$ vs $(1 / \tau) \ln \left[P_{\tau}(z) / P_{\tau}(-z)\right], \quad \tau=30,60,90 . \quad N=50, T_{L}=2.0, T_{R}=1.5$. The line labeled "Theory" corresponds to the theoretical slope $\Sigma$. The GCFT prediction agrees with the theory.

from a long-time run. Numerically we estimate $J_{\infty} \approx 0.469$, $T_{R}^{\prime} \approx 1.70 \quad($ site $n=N), T_{L}^{\prime} \approx 1.86 \quad($ site $n=1)$. Thus, $\Sigma$ $\approx 0.0237$. Figure 10 (b) clearly shows that the linear dependence on $z$ and the slope agrees with the theoretical prediction of $\Sigma$. It is surely shown that the entropy production of thermodynamics coincides with the entropy production of the GCFT.

\section{SUMMARY}

We have investigated nonequilibrium properties of the ding-dong model coupled with a heat reservoir motivated by the work of $[4,5,8,9]$. We have checked the Fourier law and entropy production in this model. Numerical calculation has shown that the GCFT holds and the entropy production is surely nonzero. Therefore, we obtain strong evidence that the GCFT is valid for the case of a heat reservoir (stochastic boundary condition). In this sense, the numerical result suggests that the GCFT may be generalized to the case of a stochastic boundary condition as was tried in [9]. For the ES identity, a careful check is needed again. We believe that the discrepancy is due to the lack of stationarity. This point will be clarified in future work.

\section{ACKNOWLEDGMENTS}

I thank K. Kitahara and S. Takesue for continuous encouragement and discussion. 
[1] P. Gaspard and G. Nicolis, Phys. Rev. Lett. 65, 1693 (1990); P. Gaspard and J. R. Dorfman, Phys. Rev. E 52, 3525 (1995);

P. Gaspard, Chaos, Scattering, and Statistical Mechanics (Cambridge Univ. Press, Cambridge, 1998).

[2] D. Ruelle, J. Stat. Phys. 85, 1 (1996); 86, 935 (1997).

[3] P. Gaspard, J. Stat. Phys. 88, 1215 (1997).

[4] G. Gallavotti and E. G. D. Cohen, Phys. Rev. Lett. 74, 2694 (1995); J. Stat. Phys. 80, 931 (1995); Doc. Math. J. DMV Extra Volume ICM 1, 65 (1998).

[5] S. Lepri, R. Livi, and A. Politi, Physica D 119, 140 (1998).

[6] J.-P. Eckmann, C.-A. Pillet, and L. Rey-Bellet, Commun. Math. Phys. 201, 657 (1999).

[7] T. Prosen and M. Robnik, J. Phys. A 25, 3449 (1992).

[8] D. Evans and D. Searles, Phys. Rev. E 50, 1994 (1994).

[9] J. L. Lebowitz and H. Spohn, J. Stat. Phys. 95, 333 (1999).

[10] J. Kurchan, J. Phys. A 31, 3719 (1998).

[11] G. Casati, J. Ford, F. Vivaldi, and W. M. Visscher, Phys. Rev.
Lett. 52, 1861 (1984).

[12] J. M. Dawson, Phys. Fluids 5, 445 (1962); Methods Comput. Phys. 9, 1 (1970).

[13] K. Kitahara, N. Ishibashi, and K. Tanno, in Some Problems on the Theory of Dynamical Systems in Applied Sciences, edited by H. Kawakami (World Scientific, Singapore, 1991), p.65.

[14] Y. Mitsumori, Bachelor's thesis, Tokyo Institute of Technology, 1993 (unpublished).

[15] A. Kubota, Master's thesis, Tokyo Institute of Technology, 1993 (unpublished).

[16] T. Yanagisawa, Master's thesis, Tokyo Institute of Technology, 1995 (unpublished).

[17] E. G. D. Cohen and G. Gallavotti, J. Stat. Phys. (to be published).

[18] R. Techver, F. Toigo, J. Koplik, and J. R. Banavar, Phys. Rev. E 57, R17 (1998). 